\title{
An FPGA Based Computer System for Greenhouse Control
}

\author{
By Nonel Thirer* \\ Igor Uchansky
}

Greenhouses provide a controlled growing environment for plants. A successful greenhouse requires the determining of many key factors, such as temperature, levels of light and shade, humidity and ventilation. Computer based systems used for data acquisition and climate control are often costly and problem prone. In this paper we present a cheap and flexible computerized system capable of controlling a greenhouse growing a strain of tomatoes popularly known as "cherry tomatoes", for its cherry-like shape (henceforth: "cherry tomatoes"). The system collects meteorological data such as light radiation, humidity, temperature and wind speed; and optimizes the climate in the greenhouse using a special algorithm that determines the movement of the greenhouses' curtains. The project's innovation lies in the use of an FPGA (Field Programmable Gate Array) technology; and an easily customized hardware-software, able to create core processors (in our case a NIOS II processor) in parallel with other logic components, by software only.

\section{Introduction}

Many projects, tests and researches have been done in order to satisfy the conditions and cultivation of crops under greenhouse. That's why it is often necessary to develop an acquisition and control system. (Mittal et al., 2012).

The evaluation of agricultural production systems is often a time consuming, costly and difficult process because it means visiting selected crop fields in greenhouses, measure and register certain physical meteorological characteristics and deciding how it affects the crop fields (Mendoza-Jasso et al., 2005).

In the acquisition and control system, the more sensors there are, the more time consuming the process is. Moreover, adding new sensors or controllersensor interaction requires adding new hardware.

In recent years, some control and monitor systems based on simple micro controllers (Mittal et al., 2012; Ameur et al., 2001) or FPGA circuits (MillanAlmaraz et al., 2013; Upasani et al., 2010; Dinesh and Saravanan, 2000; Gómez-Melendez et al., 2011) have been proposed. Many commercial data

${ }^{*}$ Senior Lecturer, HIT-Holon Institute of Technology, Israel.

${ }^{\dagger}$ B.Sc. Student, HIT-Holon Institute of Technology, Israel. 
acquisition systems such as Gavish control systems (Gavish, 2010) or CR1000 Measurement and Control System (Campbell, 2014) are also used, but their price is very high.

The objective of this research was to develop a low cost, portable, embedded computerized system for the control of a greenhouse for the growing of cherry tomatoes. The innovation of this system lies in the use of FPGA technology to create, by software, a soft core processor (in our case a NIOS II processor) and thus to use FPGA and microcontroller circuits together. Using ALTERA development FPGA kits like Cyclone V GX Starter Kit and DE0 Nano with few cheap additional parts we achieve both the low cost and the flexibility requirements.

\section{The Embedded Control System}

The embedded system (figure 1) is designed to measure and to store parameters - like temperature, humidity, light intensity and wind speed - and also to control the curtains of the greenhouse - in order to optimize the climate in the greenhouse.

The system is comprised of two low cost Altera development boards: DE0 Nano (as operator unit) and Cyclone V GX Starter Kit (as data acquisition unit).

Thus, the operator unit controls the curtains' motors, and the data acquisition unit samples all meteorological data and stores them in an SD card. The communication between the units is wireless, using low cost transceivers, whereas the data acquisition is the master unit and the operator unit is the slave unit.

\section{Figure 1. Embedded System Block Diagram}

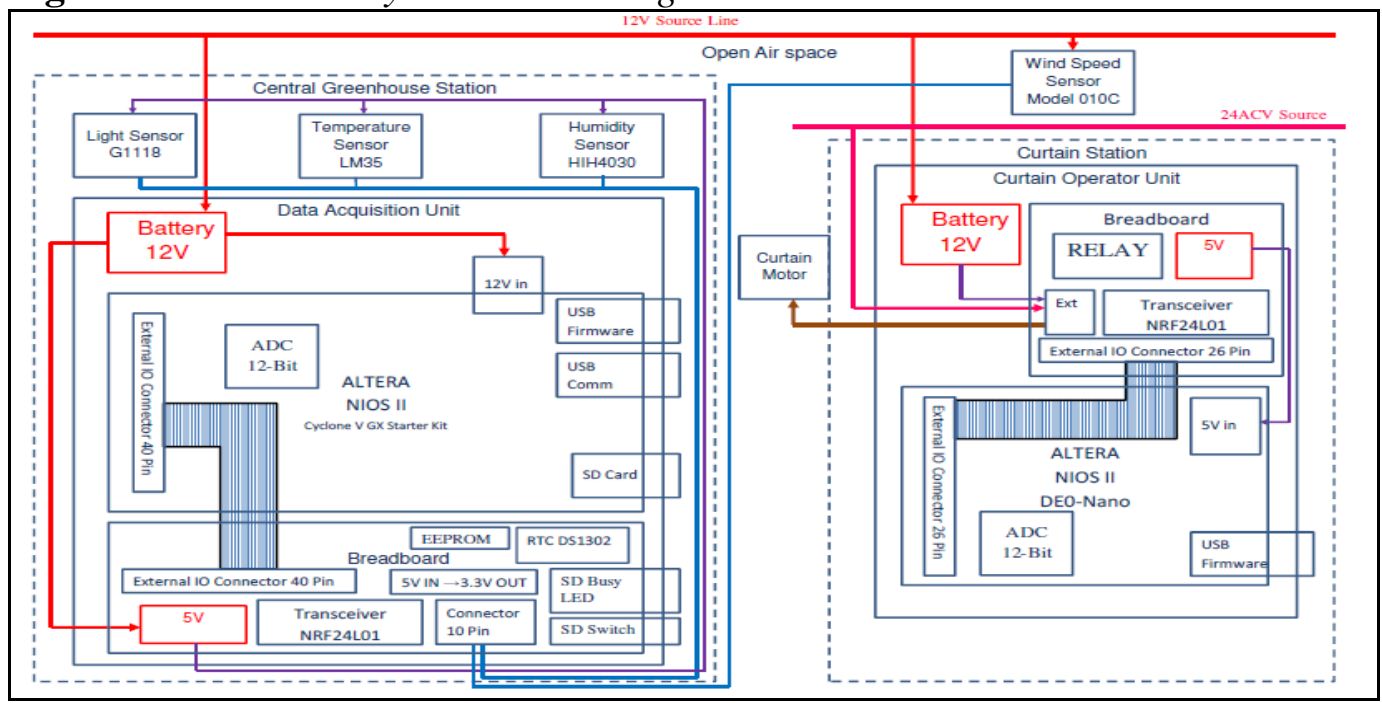

The use of both FPGA and microcontroller circuits has many advantages over using only one of them. For example, the frequency counter, counting anemometer pulses processed by FPGA and read by the soft core CPU (central 
processing unit), cancels the interrupt overhead processes which could slow down the CPU speed. Also, compiling source code for soft core CPU takes less time than synthesizing the hardware description language for the FPGA.

\section{Hardware Implementation}

\section{Data Acquisition Unit}

The data acquisition unit (figure 2) consists of a Cyclone V Starter kit board and environment sensors. The board is based on a Cyclone V FPGA and contains a $512 \mathrm{~KB}$ memory to store the CPU program code, a 12 bits analog to digital converter to sample three sensors, a bridge for establishing connection to PC, a flash memory to store the FPGA configuration and program code, and also a micro SD card connection for storing sensors samples. The implemented NIOS II CPU is a 32 bits RISC microprocessor. All CPU connections to its peripherals are made through a special environment which generates the HDL (hardware development language) code for them and thus the drivers are automatically generated. Basically, ALTERA provides the common generic IP (Intellectual Property) cores (as UART and SPI interfaces, SRAM memory, timers etc.) and if a new IP core is required it can be built from scratch or found at commercial products. A special IP Core was built for every environment sensor: the temperature sensor (LM35) - which provides a linear output voltage $\left(10 \mathrm{mV} /{ }^{\circ} \mathrm{C}\right)$ in the is $0^{\circ} \mathrm{C}$ to $100^{\circ} \mathrm{C}$ range; the humidity sensor (HIH4030) - with a fast response and a quasi linear output in the $0 \%$ to $100 \%$ relative humidity $(\mathrm{RH})$ scale; the light intensity sensor (G1118) - with an output current (for a $100 \Omega$ load) between $10^{-3} \mathrm{~A}$ to $10^{-14} \mathrm{~A}$ for a $10^{-2} \mathrm{~W}$ to $10^{-13}$ $\mathrm{w}$ radiation; a three cups anemometer $(010 \mathrm{C})$ - which provides an accurate response for horizontal wind speed in the $0-125 \mathrm{mph}(0-60 \mathrm{~m} / \mathrm{s})$ range.

Figure 2. Data Acquisition Unit IP Cores

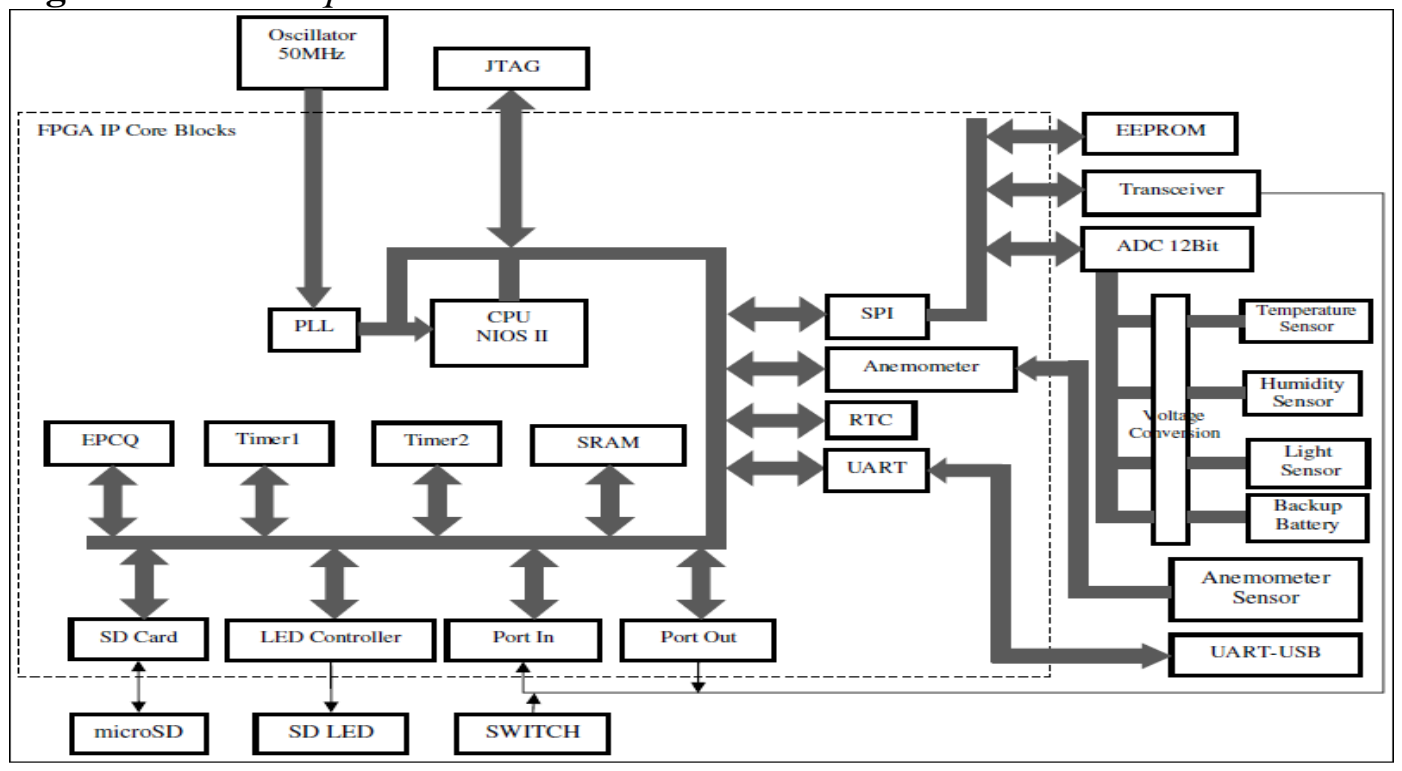




\section{Curtain Operator Unit}

The Curtain operator unit (figure 3) consists of a DE0 Nano board, based on a Cyclone IV FPGA chip. The board contains $32 \mathrm{MB}$ memory to store the CPU program code, a 12 bits analog to digital converter to sample the battery backup voltage and a flash memory to store the FPGA configuration and program code.

Figure 3. Curtain Operator Unit IP Cores

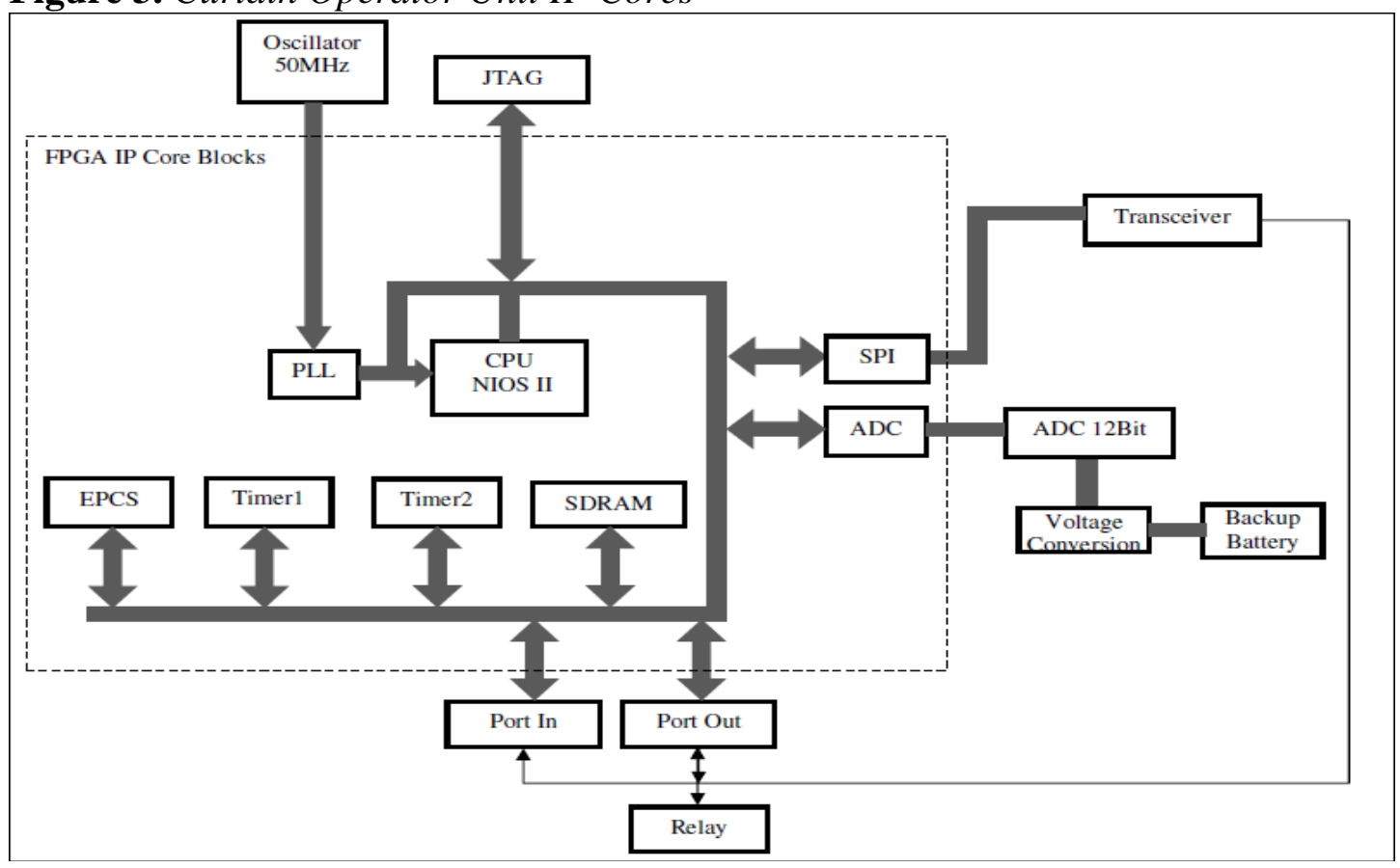

\section{Software Implementation}

The software of the greenhouse monitoring and controlling system is based on the $\mathrm{C}$ programming language for the CPU NIOS II, and on the C\# programming language for a friendly graphical user interface (GUI).

\section{Algorithm}

The following chart (figure 4) presents the greenhouse measurement and curtain control algorithm.

The measurement sensors are sampled every 10 seconds and the average is done every 5 minutes for a real time update of the SD card. If the SD card switch is pressed for less than 3 seconds, the SD card can be injected/ inserted, otherwise the unit enters PC mode. Pressing this switch again, makes the unit return back to normal mode. 
Figure 4. CPU Software Flow Chart

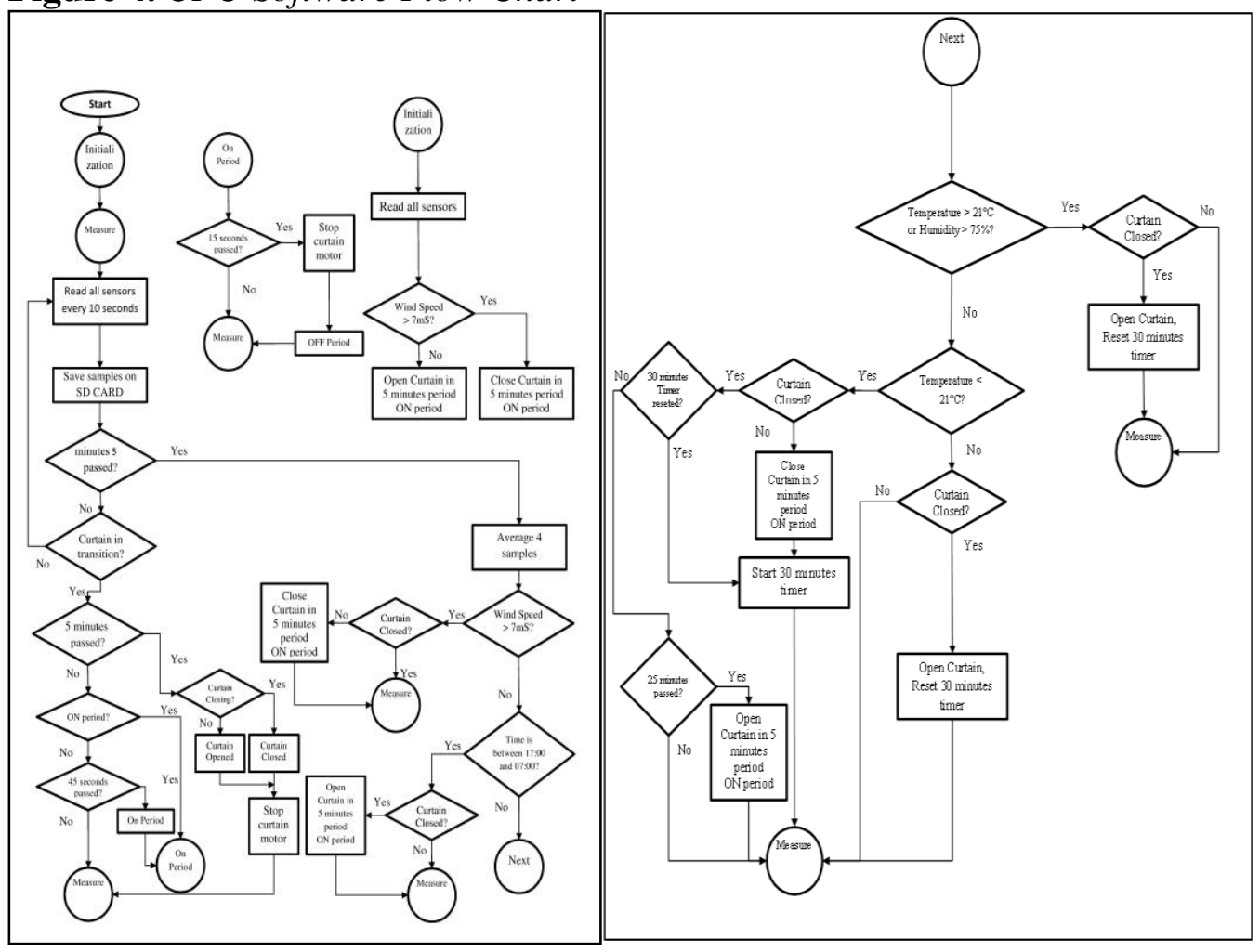

The priorities to close or to open the curtains are as defined:

- When there is a sand storm (the wind speed is at least $7 \mathrm{~m} / \mathrm{s}$ ), all curtains must be closed.

- At night, defined by the real time clock (RTC) or by a low light intensity, all curtains must be opened.

- If the temperature is at least $21^{\circ} \mathrm{C}$ or the humidity is more than $75 \%$, all the curtains must be open.

- If the temperature is less than $21^{\circ} \mathrm{C}$, periodically all the curtains must be close for 25 minutes and open for 5 minutes.

To open/ close the curtains a step by step progressive process is also defined: in the first 15 seconds the motor works to open/close the curtain and in the next 45 seconds it will stop, a process which is repeated five times.

\section{The GUI Interface}

The graphic user interface (figure 5) is designed to monitor, configure and debug the whole data acquisition and curtain operator units. It permits to configure and update the system, to read the sensor measurements and batteries status, to open/close manually the curtain and also to use the SD card. 
Figure 5. GUI Interface

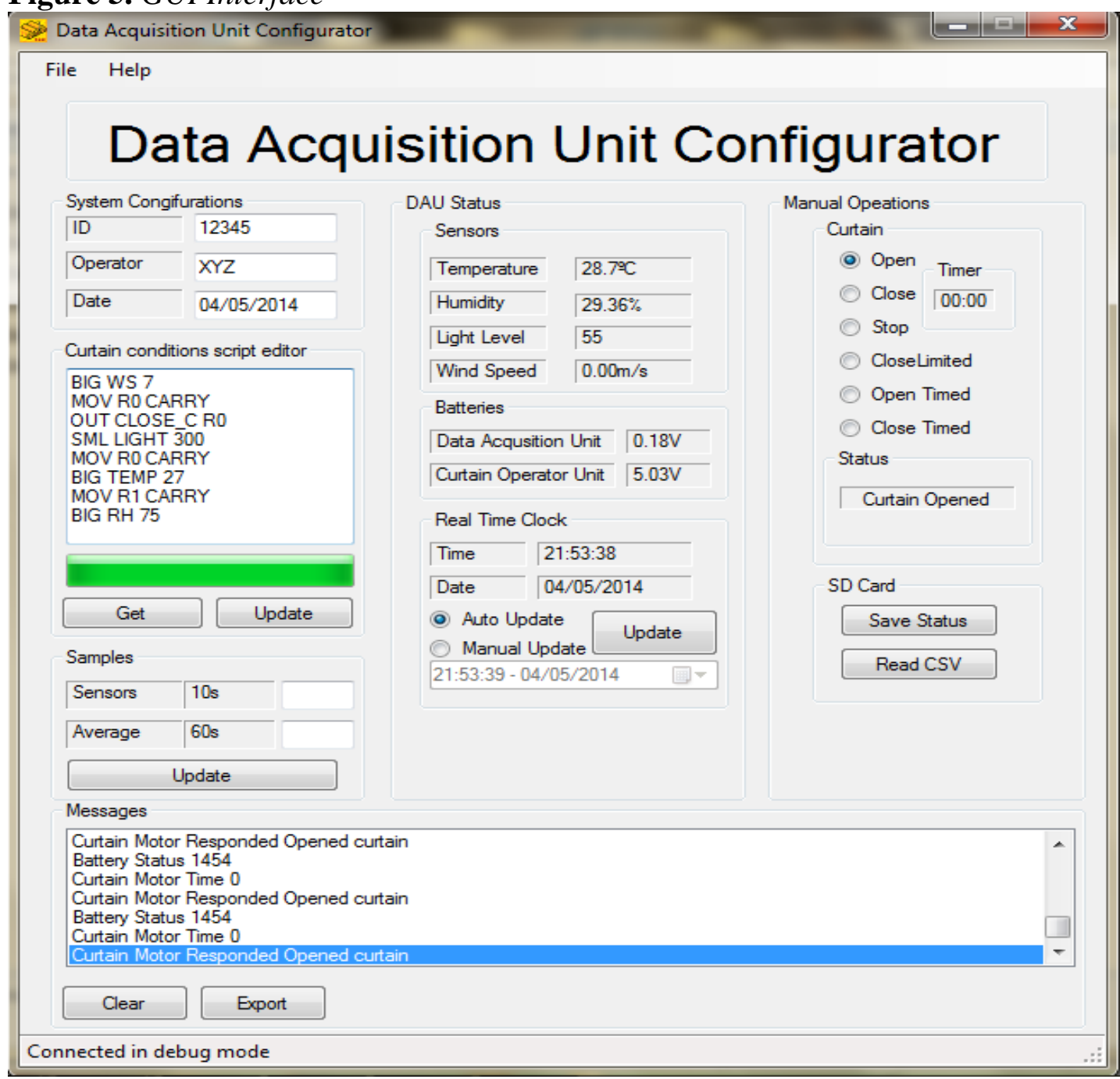

\section{Experimental Results}

The temperature, humidity and light sensors were set close to each other, and sampled the environment. The first configuration was sampled every 10 seconds, and averaged every 5 minutes.

For system testing, the conditions were set to be:

- Close curtain if wind speed $>7 \mathrm{~m} / \mathrm{s}$

- Open curtain if time $<07: 00$ or time $>20: 00$ or humidity $>85 \%$ or temperature $>27^{\circ} \mathrm{C}$

- Close curtain for limited time (a 30 minutes cycle : 25 minutes close and 5 minutes open) if temperature $<24^{\circ} \mathrm{C}$

The results presented below are after one day running and after one week running. The data was stored on line on a SD card. 
Figure 6. One Day Running

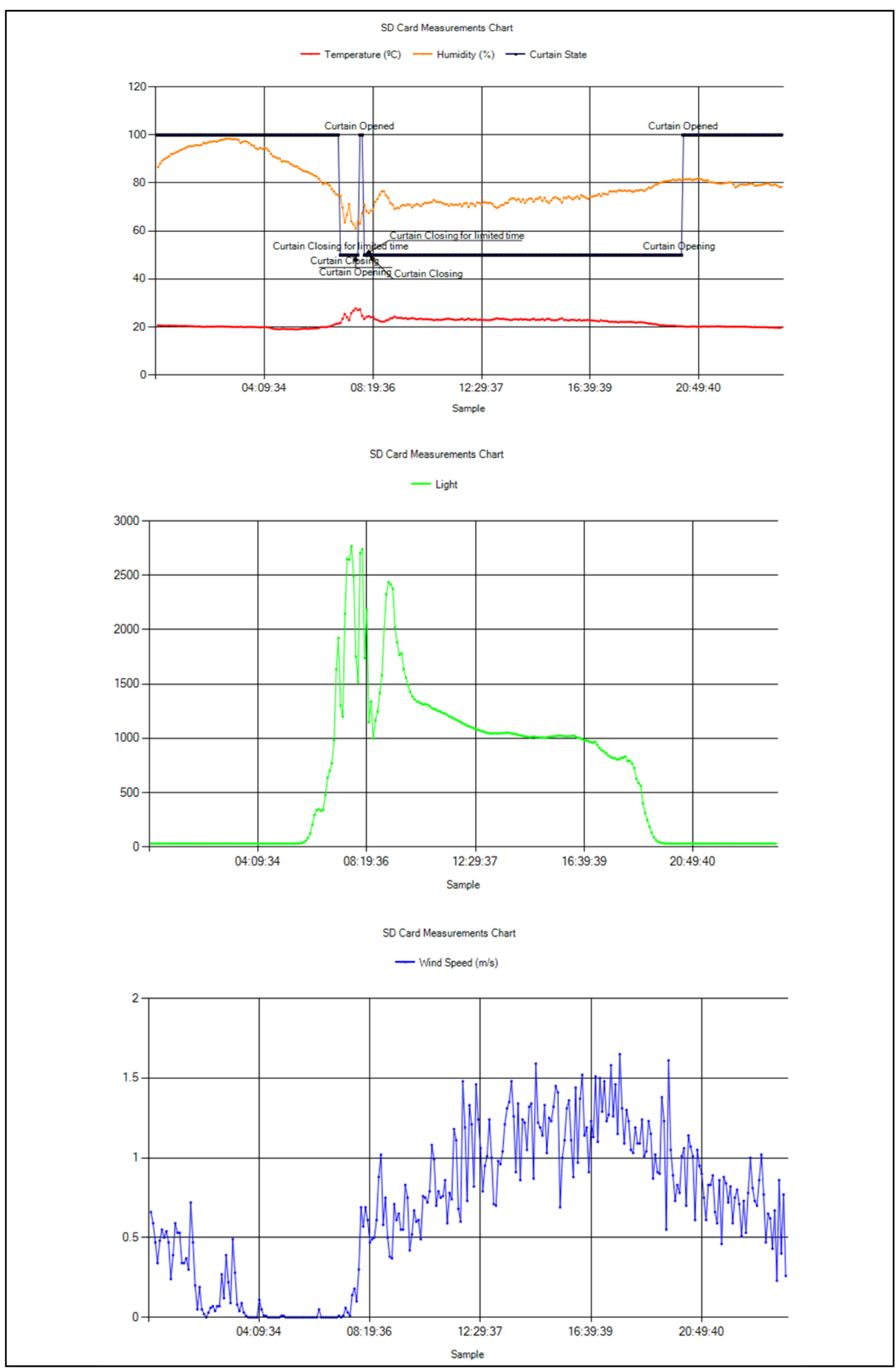




\section{One Day Running}

As shown in figure 6, when the temperature rises, the humidity falls respectively. Also the light rises as sun rises near 06:00 and falls as we near 19:00 o'clock. Most wind appears between 13:00 and 16:00. Curtain opening conditions are met when the time is less than 07:00 or more than 20:00, the rest of the time it is opened for limited periods because at first the temperature is below $24{ }^{\circ} \mathrm{C}$ and after that the temperature rises above $27^{\circ} \mathrm{C}$ for a short period which triggers to open the curtain; but later, the temperature drops again below $24^{\circ} \mathrm{C}$ and the curtain closes again for a limited period. The curtain was never closed for longer time because the wind speed condition was never met.

\section{One Week Running}

The system ran for a week. The results are shown below (figure 7).

Figure 7. One Week Running

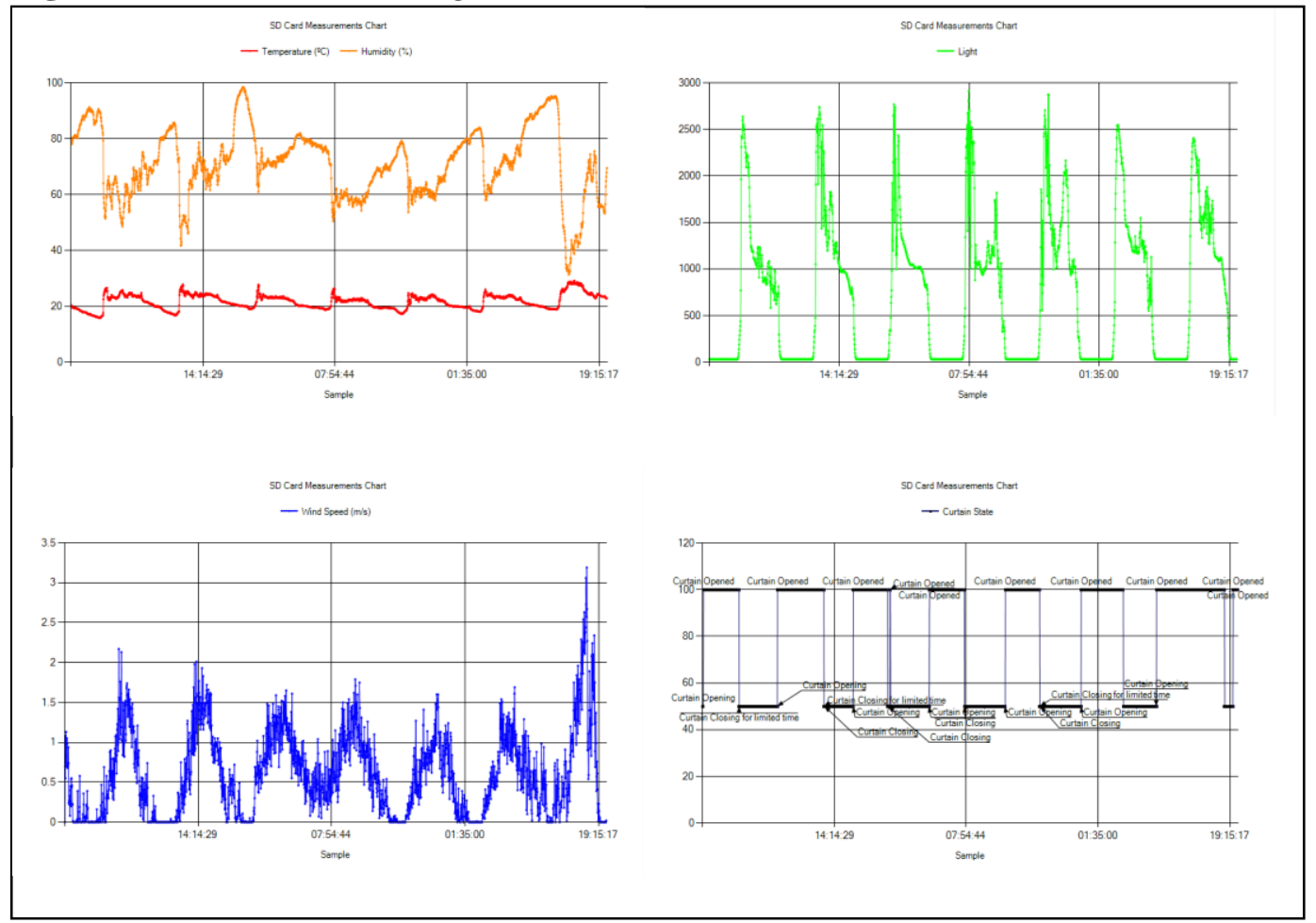

As seen in figure 7, the measurements of temperature, humidity, light and wind speed are done periodically. The temperature is higher at day time but lower at night. The night temperature tends to rise every day; wind is mostly present around noon. The curtains are also open and closed in accordance with the required algorithm. 


\section{Conclusions}

Using the FPGA technologies, an embedded acquisition and control system was developed. The results obtained from the data acquisition unit and also from the curtain operator unit demonstrate that the system's performance is accurate and reliable. The system's installation is easy and requires only minimal electronic knowledge, being similar to other data loggers for weather stations.

The system has successfully proved its superiority over existing systems by reducing the cost while at the same time increasing versatility. New sensors can be added in the future. Also, a connection to a GSM modem is possible, since at the moment the logic power of the FPGA circuits is only used at around $10 \%$ in the acquisition unit and $22 \%$ in the curtain operator unit.

\section{Acknowledgments}

The authors would like to thank Dr. Shabtai Cohen, researcher at the Volcani Center for his most valuable and precious assistance and consulting.

\section{References}

Ameur S., Laghrouche M., Adane A., 2001, Monitoring a greenhouse using a microcontroller-based meteorological data acquisition system, Renewable Energy, Volume 24, Issue 1, September 2001, 19-30.

Campbell, 2014, Campbell Scientific Inc., Available at http://www.campbellsci.com

Dinesh M., Saravanan P., 2000, FPGA Based Real Time Monitoring System for Agricultural Field, International Journal of Electronics and Computer Science Engineering, Volume 1, No 3, (2000), 1514-1519.

Gavish, 2010, Gavish Control systems Ltd., Available at http://www.gavish.org.il

Gómez-Melendez D., López-Lambraño A., Herrera- Ruiz G., Fuentes C., Rico-Garcia E., Olvera-Olvera C., Alaniz-Lumbrerasc D., Mercado Fernández T. and Verlinden S., 2011, Fuzzy irrigation greenhouse control system based on a field programmable gate array, African Journal of Agricultural Research, Vol. 6(13), (4 July, 2011), 3117-3130.

Mendoza-Jasso J., Ornelas-Vargas G., Castãneda-Miranda R., Ventura-Ramos E., Zepeda-Garrido A., Herrera-Ruiz G., 2005, FPGA-based real-time remote monitoring system, Computers and Electronics in Agriculture 49 (2005), 272285.

Millan-Almaraz J.R., Torres-Pacheco I., Duarte-Galvan C., Guevara-Gonzalez R.G., Contreras-Medina L.M., de Jesus Romero-Troncoso R., Rivera-Guillen J.R., 2013, FPGA-based wireless smart sensor for real-time photosynthesis, Computers and Electronics in Agriculture 95 (2013), 58-69.

Mittal M., Tripathi G., Chauhan D., Agarwal A., 2012, Green House Monitor and Control Using Wireless System Network, VSRD-IJEECE, Vol. 2 6, (2012), 337345. 
Vol. 2, No. 1 Thirer et al.: An FPGA Based Computer System...

Upasani D.E., Shrote S.B., Wani V.P., 2010, FPGA Implementation of intelligent climate control for greenhouse, International Journal of Computer Applications (0975 - 8887) Volume 1, No. 18, (2010), 17-21. 